\title{
Multimode Horn Antennas for Far-Infrared Astronomy
}

\author{
J. A. Murphy ${ }^{1}$, C. Bracken ${ }^{1}$, S. Doherty ${ }^{1}$, I McAuley ${ }^{1}$, D Wilson ${ }^{1}$, C. O'Sullivan ${ }^{1}$, N. Trappe ${ }^{1}$, M. Gradziel ${ }^{1}$, P. Ade ${ }^{2}$, \\ E. Gleeson ${ }^{1}$, B Maffei $^{3}$, J-M Lamarre ${ }^{4}$, F. Noviello ${ }^{3}$, T Peacocke ${ }^{1}$, G Pisano ${ }^{3}$, J-L Puget ${ }^{5}$, G Savini ${ }^{6}$, R. Sudiwala ${ }^{2}$, S. \\ Withington $^{7}$, R. Wylde ${ }^{8}$, V Yurchenko ${ }^{1}$ \\ ${ }^{1}$ Experimental Physics Department, National University of Ireland Maynooth, Maynooth, Co. Kildare, Ireland. \\ ${ }^{2}$ Cardiff School of Physics and Astronomy, Cardiff University, Queen's Buildings, The Parade, Cardiff CF24 3AA, UK \\ 3 Jodrell Bank Centre for Astrophysics, School of Physics and Astronomy, Oxford Road, Manchester M13 9PL, UK \\ ${ }^{4}$ Lerma, Observatoire de Paris, 61 avenue de l'Observatoire, 75014 Paris, France \\ ${ }^{5}$ Institut d'Astrophysique Spatiale, Universite de Paris XI, bat. 121, 91405 Orsay Cedex, France \\ ${ }^{6}$ Department of Physics \& Astronomy, UCL, Kathleen Lonsdale Building, Gower Place, London WC1E 6BT, UK \\ ${ }^{7}$ Cavendish Laboratory, University of Cambridge, JJ Thomson Avenue, Cambridge CB3 OHE, UK \\ ${ }^{8}$ St Andrews University, School of Physics and Astronomy, North Haugh, St Andrews, Fife KY16 9SS, Scotland, UK
}

\begin{abstract}
Multi-mode horns combined with bolometric or incoherent detectors are finding application in astronomical receivers for which partially coherent operation can provide increased throughput and thus sensitivity. This is advantageous when observing faint sources, especially if diffraction limited resolution is not required, or if the horn beam is truncated by a cold stop in the optical train. We discuss how such horns can be simulated and present examples from receiver instrumentation on the Planck and SPICA space telescopes.
\end{abstract}

Index Terms-horn antenna, multimode, far-infrared astronomy.

\section{InTRODUCTION - MUlTimOde HornS}

In telescope systems for far-infrared astronomy multimode horn antennas provide many of the same advantages as single mode horns in coherent systems particularly for the control of beam pattern characteristics - the difference being that the typical waveguide structure feeding the multimode horn is oversized and allows higher order modes to propagate, all of which can carry extra power to the detector. In contrast to a coherent system these modes couple independently to the detector providing controlled beam definition with the further advantage of higher filling factors for array operation.

In the European Space Agency Planck satellite, which has mapped the anisotropies of the cosmic microwave background radiation with unprecedented sensitivity and high angular resolution [1], such multimode horns were utilized in the two highest frequency non-CMB $(545 \mathrm{GHz}$ and $857 \mathrm{GHz})$ channels of the HFI instrument to allow for the robust removal of foreground sources from the data [2]. The requirement for high sensitivity and similar resolution to the CMB bands, while still requiring exquisite control of beam pattern characteristics at the telescope mirrors, was an ideal application for the multimode horn systems.

In Planck these horns were corrugated and configured in a back-to-back setup ,which transmitted the signal through filters to a detector horn feeding a cavity mounted bolometer. Simulations of the complex interactions of the horns was computationally challenging especially when the complex behavior across the $30 \%$ bandwidth of the channels was included in the analysis, with the prediction of the broadband beam patterns on the sky also requiring careful consideration [3]-[7].

Similarly multimode horn antennas are now also being investigated as potential feeds for a TES detector array for the SAFARI instrument on the SPICA satellite [8]. In this case profiled smooth walled horns are being proposed, which increases the level of complexity for optimizing the sensitivity of the system as a cold stop in the far field of the horn array actually defines the point spread function at the array, which is large enough to couple simultaneously to several horns. The horns plus detectors effectively behave as pixels in the same way as bare bolometer arrays, except the beams are controlled and coupling to the background thermal noise is minimized.

\section{ELECTROMAGNETIC MODELLING}

Waveguide fed multimode horn antennas can be modeled most efficiently using a scattering matrix technique, where the waveguide entrance port is excited by a number of independent modes and not just a single coherent mode as is the case of single-mode horns. For cylindrically symmetric horns this implies that higher azimuthal order $\mathrm{n}=1$ modes, as well as orders for which $\mathrm{n} \neq 1$, can in principle also propagate depending on their cut-off properties in the waveguide. If the cylindrical guides are corrugated (as in the PLANCK example discussed above) then hybrid $\mathrm{HE}$ and $\mathrm{EH}$ modes (combinations of TE and TM fields) become the true modes of propagation [9]. Similarly for an overmoded rectangular guide, as is being proposed for SAFARI, modes other than the fundamental $\mathrm{TE}_{10}$ can clearly also propagate and also independently couple to the incoherent detectors [10],[11]. 
The problem can be simplified if the overmoded waveguide effectively feeds a quasi-blackbody integrating cavity, in which the bolometer is mounted. Then reciprocity can be applied and all waveguide modes at the waveguide port can be considered to be excited independently of each other with no phase relationship between them. Thus, many modes will contribute incoherently to the beam on the sky radiated by the horn. The case of a smooth walled rectangular waveguide is simpler than a corrugated feed, as there is no scattering within the waveguide itself, although there will be scattering in the horn (especially a profiled horn) and thus the problem is best dealt with using mode matching software. In fact, profiled shaped horns have been used on Planck and are being proposed for SAFARI also (see Fig. 1).

In the modeling such profiled horns are regarded as a sequence of very short smooth-walled waveguide sections (representing sequentially either the corrugation grooves and fins for corrugated systems or as steps of a stair-like profile for smooth walled designs). At the junction between two segments scattering of power between modes can occur, which is kept track of using a mode matching technique such that complex power is conserved. Thus, one can compute the cascaded scattering matrix for complete shaped waveguide horn structures. The details of the electromagnetic theory underlying multimode horn operation and the use of this scattering matrix approach is described in a number of publications [10],[12]. The mode matching approach has been shown to predict the beam patterns of single mode horns to high accuracy and reproducibility [7].

It is worth pointing out that in this discussion the term "mode" can be ambiguous, being both used within the context of hybrid modes and the mode matching scattering matrix technique, in which smooth-wall waveguide modes act as the "basis" set of fields.

\section{BEAM PATTERN PREDICTIONS}

At the front aperture of the horn one can recover, using SVD analysis, the true independent orthogonal fields transmitted by the waveguide filter and horn that constitute the partially coherent beam. These fields propagate independently through the telescope and onto the sky, since they were generated by an independent set of waveguide fields at the effective black body enclosure at the detector [10]. The columns of the $S_{21}$ (in the usual notation) scattering matrix contain the mode coefficients for the modal sums required to recover these independent hybrid fields at the horn aperture.

These fields, once recovered, must be propagated to the sky independently and then be added in quadrature to simulate a true multimode beam pattern, each simulation being at a single frequency. In order to produce a true broad band beam these single frequency intensity patterns must then furthermore be added across the band with non-uniform weighting to take into account any non-uniform transmission for the filters that define the band. The spectrum of the source will also affect the power coupled for any particular observation.

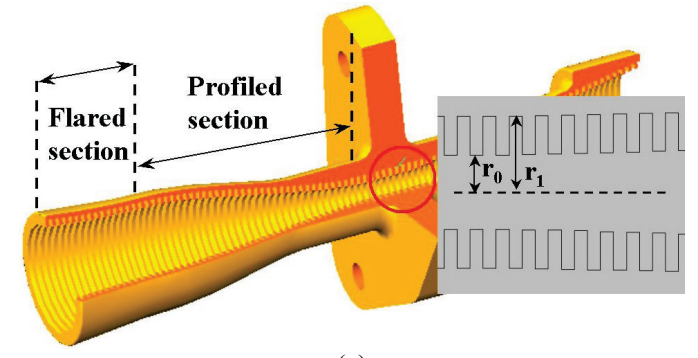

(a)

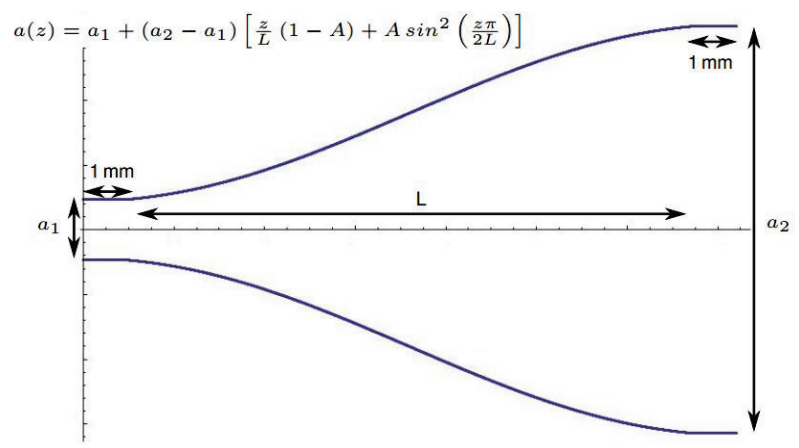

(b)

Fig. 1. (a) Corrugated profiled horns used on Planck-HFI (above) - see Ref. [2] and (b) the profiled square smooth walled horns proposed for SAFARI (below)

As indicated above it is a reasonable approximation to assume that cavity acts as a radiator. However, it is possible to also model the horn waveguide cavity detector system as an absorber and use the $S_{11}$ reflection scatter matrix, as seen by an incoming wave from infinity for the simulations of the telescope beam patterns on the sky described. Any non-ideal black body behavior of the cavity can then be simulated. This approach is being pursued in the SAFARI horn simulations.

The plots below in Fig. 2 are the predicted far field patterns for both a Planck HFI horn and a SAFARI pixel. The SAFARI pixel uses a square cross section profiled horn and it was modeled as an absorbing system, in which the $S_{11}$ scattering matrix indicated for which directions in the sky incident power was most efficiently absorbed.

\section{CONCLUSION}

We have summarized the approach to the design and modeling of multi-mode horns for far-infrared astronomy.

With this approach the optical requirements on the performance of the horns are met in terms throughput, illumination of the telescope exit aperture and beam patterns on the sky.

We illustrated the basic principles of multi-mode horn operation with examples from the PLANCK and SPICA space telescopes. 


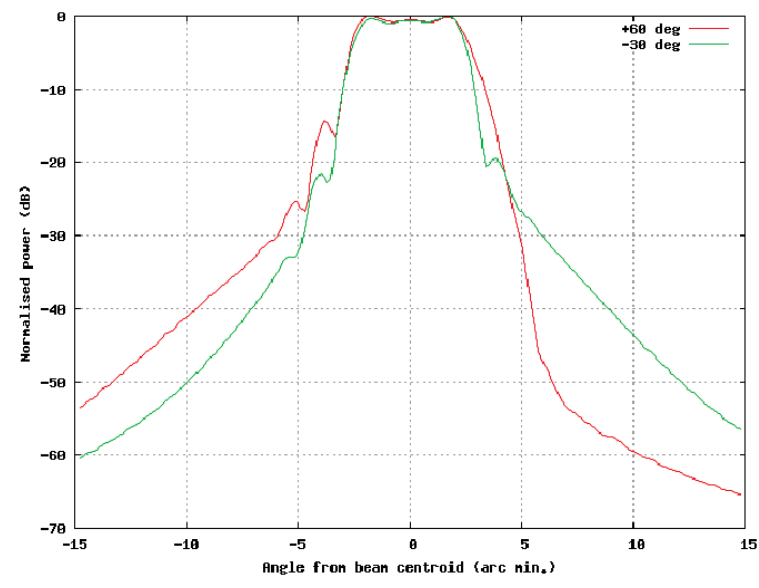

(a)

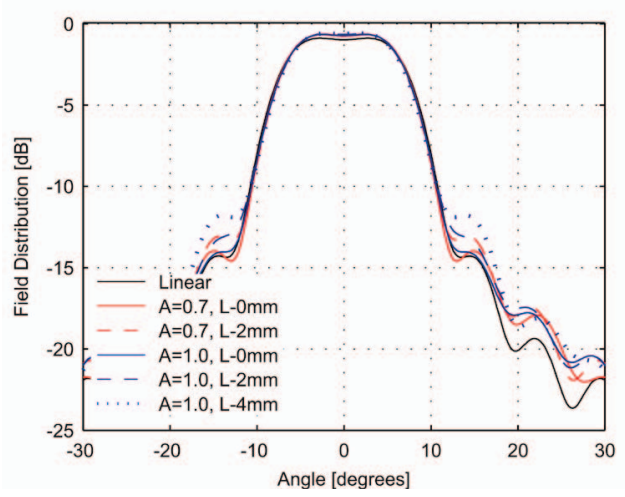

(b)

Fig. 2. Simulated far-field beam patterns of (a) Planck-HFI $857 \mathrm{GHz}$ multimode horn as reported in Ref. [2] (upper plot) and (b) proposed SAFARI Lband profiled multi-mode horn (lower plot).

\section{ACKNOWLEDGMENT}

The authors would like to acknowledge support for this research from the European Space Agency and Enterprise Ireland through PRODEX and TRP contract funding.

\section{REFERENCES}

[1] Ade et al., "Planck 2013 results. I. Overview of products and scientific results," unpublished.

[2] J A Murphy et al., "Multi-mode horn design and beam characteristics for the Planck satellite," Journal of Instrumentation, vol. 5, T04001, April 2010

[3] J. Tauber et al., "Planck pre-launch status: the Planck mission," A\&A, vol. 520, A1, September 2010

[4] J. Tauber et al., "Planck pre-launch status: the optical system of Planck," A\&A, vol. 520, A2, September 2010

[5] J-M. Lamarre et al., "Planck pre-launch status: the HFI instrument," A\&A, vol. 520, A9, September 2010

[6] P.A.R. Ade et al., "Planck pre-launch status: the optical architecture of the HFI," A\&A, vol. 520, A11, September 2010

[7] B. Maffei et al., "Planck pre-launch status: HFI beam expectations from the optical optimisation of the focal plane, " A\&A, vol. 520, A15, September 2010

[8] Trappe et al. "Optical modeling of waveguide coupled TES detectors towards the SAFARI instrument for SPICA," Proceedings of SPIE, vol. 8452, 84520L, August 2012

[9] A.D. Olver, P.J.B. Clarricoats, A.A. Kishk and L Shafai., Microwave horns and feeds, New York:IEEE Press, 1994.

[10] J.A. Murphy, R. Colgan, C. O’Sullivan, B. Maffei and Peter Ade, "Radiation patterns of multi-mode corrugated horns for far-IR space applications," Infrared Physics \& Technology, vol. 42, pp. 515-528, December 2001

[11] S. Withington and J.A. Murphy, Modal analysis of partially coherent submillimeter-wave quasi-optical systems", IEEE Trans Antennas \& Propagat, vol. 46, 1651-1659, November 1998

[12] E. Gleeson et al., "Corrugated waveguide band edge filters for CMB experiments in the far infrared, "Infrared Physics \& Technology, vol. 46, pp. 493-505, August 2005 